\title{
Utilidad del Gastroschisis Prognostic Score (GPS) para predecir la estancia intrahospitalaria en los recién nacidos
}

\section{Usefulness of Gastroschisis Prognostic Score (GPS) to predict the intrahospital length of stay in newborns}

\author{
Manuel Gil-Vargas ${ }^{1 *}$, Agustín Gutiérrez-Urióstegui², Elvia P. Concha-Ramírez², \\ Mariana L. Miguel-Sardaneta ${ }^{3}$, Estefanía Zepeda-Muñoz ${ }^{4}$ y Mary S. Saavedra-Pacheco ${ }^{5}$
}

${ }^{1}$ Servicio de Cirugía Pediátrica, Hospital General de Puebla Eduardo Vázquez Navarro, Servicios de Salud del Estado de Puebla; ${ }^{2}$ Servicio de Neonatología, Hospital para el niño Poblano, Servicios de Salud del Estado de Puebla; ${ }^{3}$ Servicio de Pediatría, Hospital General Zona Norte, Servicios de Salud del Estado de Puebla; ${ }^{4}$ Facultad de Medicina, Universidad de las Américas de Puebla; ${ }^{5}$ Facultad de Medicina, Benemérita Universidad Autónoma de Puebla. Puebla, México

\section{Resumen}

Antecedentes: La gastrosquisis es un defecto congénito caracterizado por la evisceración de órganos a través de la pared abdominal. El Gastroschisis Prognostic Score (GPS), escala de daño intestinal, es una herramienta que permite identificar a pacientes con bajo o alto riesgo de morbilidad y mortalidad. Objetivo: Evaluar la utilidad de la puntuación del GPS de los recién nacidos para predecir la estancia intrahospitalaria. Material y métodos: Estudio observacional, transversal y retrospectivo llevado a cabo en cuatro centros hospitalarios de marzo de 2014 a diciembre de 2019. Se estudiaron las siguientes variables: edad en horas a la 1. ${ }^{a}$ cirugía, edad en días a la 2. ${ }^{a}$ cirugía, días de estancia intrahospitalaria y complicaciones. Resultados: 83 recién nacidos con gastrosquisis fueron divididos en los que evolucionaron adecuadamente, los que presentaron morbilidades y los que fallecieron. Se estratificaron en cuatro subgrupos de acuerdo con el GPS. Este puntaje con las complicaciones desarrolladas presentó una $p<0.05$, así como con la asociación de las causas de mortalidad. Conclusiones: Un puntaje menor a 5 predice mayor estancia intrahospitalaria, mientras que en el grupo de mortalidad y en los pacientes que evolucionaron de forma favorable no se demostró significancia estadística.

Palabras clave: Gastrosquisis. Escala de daño intestinal. Pronóstico. Duración de la estancia hospitalaria.

\section{Abstract}

Background: The gastroschisis is a congenital defect that results from herniation of the intestines through a right-sided abdominal wall defect. The Gastroschisis Prognostic Score (GPS) is a tool that allows us to identify a patient with low or high risk of morbidity and mortality. Objective: To assess the usefulness of the GPS of newborns to predict hospital length stay. Methods: Observational, cross-sectional and retrospective study carried out in 4 hospital centers from March 2014 to December 2019. The following variables were studied: age in hours at $1^{\text {st }}$ surgery, age in days at $2^{\text {nd }}$ surgery, length of stay in days and added complications. Results: 83 newborns with gastroschisis were divided into those with adequate progress, those with morbidities, and those who died. They were stratified into 4 subgroups according to the GPS score. This score with the complications developed presented a $p<0.05$, likewise with the association of the causes of mortality.

\section{Correspondencia:}

*Manuel Gil-Vargas

E-mail: gilvm@yahoo.com
Disponible en internet: 09-04-2021 Rev Hosp Jua Mex. 2021;88(1):22-26

www.revistahospitaljuarez.com 1405-9622/@ 2020 Sociedad Médico-Quirúrgica del Hospital Juárez de México, A.C. Publicado por Permanyer. Este es un artículo open access bajo la licencia CC BY-NC-ND (http://creativecommons.org/licenses/by-nc-nd/4.0/). 
Conclusion: This study is one of the few studies in the literature that shows a direct comparison between the score obtained from the GPS and the days of hospital length stay.

Key words: Gastroschisis. Gastroschisis Prognostic Score. Prognostic. Length of hospital stay.

\section{Introducción}

La gastrosquisis es un defecto congénito caracterizado por la evisceración de órganos a través de la pared abdominal, adyacente y usualmente a la derecha de la inserción del cordón umbilical. Actualmente su frecuencia va en aumento. En México, del 2000 al 2014, la incidencia aumentó de 2.09 a 6.85 por cada 10,000 nacimientos. En países subdesarrollados su mortalidad es mayor del $15 \%$, por lo general consecuente a sepsis ${ }^{1,2}$.

El Gastroschisis Prognostic Score (GPS), escala de daño intestinal, fue desarrollado por Cowan, et al. ${ }^{3}$. Es una herramienta de puntuación que se realiza poco después del nacimiento de un paciente con gastrosquisis. Permite identificar a pacientes con bajo $(<2) 0$ alto $(\geq 2)$ riesgo de morbilidad y mortalidad con base en la presencia y la gravedad de cuatro parámetros: necrosis, adherencias, atresia y perforación intestinal.

El impacto económico que desencadena una patología como la gastrosquisis en las unidades médicas es elevado, desde medicamentos y monitorización durante la estancia intrahospitalaria (EIH) hasta el uso de cuidados intensivos neonatales. En México y otros países de América Latina, las malformaciones congénitas se encuentran dentro de las principales causas de morbilidad y mortalidad en la infancia ${ }^{4,5}$, por lo que una evaluación temprana resulta de gran importancia para poder detectar posibles complicaciones y no prolongar la EIH de manera innecesaria.

El objetivo es evaluar la utilidad del GPS de los recién nacidos para predecir la ElH.

\section{Materiales y métodos}

Se trató de un estudio observacional, transversal y retrospectivo que se llevó acabo con base en una revisión de expedientes de recién nacidos con diagnóstico de gastrosquisis que fueron evaluados con el GPS. Se estudiaron 83 recién nacidos, atendidos en el periodo comprendido de marzo de 2014 a diciembre de 2019 en el Hospital General de Puebla Dr. Eduardo Vázquez Navarro, el Hospital de la Mujer Puebla, el Hospital General de Puebla Zona Norte y el Hospital del Niño Poblano.
Los criterios de selección para estos pacientes fueron los siguientes: se incluyeron a todos los recién nacidos con gastrosquisis y se excluyeron a todos los recién nacidos con expediente clínico incompleto y pacientes cuyos padres no otorgasen su consentimiento para entrar al protocolo de estudio. Se eliminaron a todos los recién nacidos que ingresaron a las unidades hospitalarias y que fueron a otro centro para su recuperación postoperatoria. Las variables estudiadas fueron: semanas de gestación, edad en horas a la 1. ${ }^{\text {a }}$ cirugía, edad en días a la $2 .{ }^{a}$ cirugía y días de ElH.

El presente estudio se realizó siguiendo los lineamientos éticos de la investigación en seres humanos indicados en la Declaración de Helsinki, el Código de Núremberg, el Informe Belmont, las Normas CIOMS y las Buenas Prácticas Clínicas para las Américas, así como de acuerdo con el Reglamento de la Ley General de Salud, en particular el artículo 13, (concerniente al respeto, dignidad y protección de los derechos del paciente pediátrico), el artículo 17 (respecto al cual se consideró una investigación de riesgo mínimo), el 20 y el 21 (que regulan el consentimiento informado). La investigación también se circunscribió a las indicaciones de la Norma Oficial Mexicana NOM-012-SS3-2012 para la ejecución de proyectos de investigación para la salud en seres humanos. Todos los padres de los pacientes que aceptaron participar en el protocolo firmaron una carta de consentimiento previa información de los objetivos de la investigación.

Este estudio de investigación fue aprobado por el comité de ética e investigación de las diferentes unidades hospitalarias.

Se analizaron las variables en una base de datos en el programa de estadística SPSS versión 25. Las variables discretas se expresaron como frecuencia y porcentaje y para las variables continuas se expresaron como media y desviación estándar (DE). Para la asociación de variables categóricas ocupamos la ji cuadrada o prueba exacta de Fischer cuando la primera no fue posible. En el análisis de tres grupos independientes buscamos inicialmente pruebas de normalidad y dado que esta no se cumplió, empleamos estadística no paramétrica, con la prueba de Kruskal-Wallis. Para demostrar la relación existente entre una variable 
cuantitativa y una variable dependiente ocupamos regresión lineal múltiple.

\section{Resultados}

El estudio se realizó entre marzo de 2014 y diciembre de 2019 con una muestra de 83 recién nacidos con el diagnóstico de gastrosquisis. De los 83 fallecieron 7 . El promedio de las semanas de gestación fue de 36.25 , con una DE de 1.93 y un rango de 32-40. En la variable edad a la $1 .{ }^{a}$ cirugía se obtuvo una media de 41.05 horas, con una DE de 44.71 y un rango de 1-192, mientras que en la edad a la $2{ }^{a}$ cirugía fueron 4 días, 4.71 y $0-21$, respectivamente.

En los que se realizó cierre primario del defecto, 26 de ellos presentaron alguna complicación (72\%), cuatro pacientes murieron (11\%) y seis no sufrieron ninguna complicación (17\%). En el caso de los pacientes que tuvieron dos cirugías para el cierre de la pared abdominal, 34 tuvieron complicaciones (72\%), tres murieron (6\%) y diez pacientes no tuvieron ninguna complicación (22\%).

Se estratificó a los pacientes con cierre primario y con cierre en dos tiempos de acuerdo con las causas de morbilidad. En los pacientes con cierre primario, 20 de ellos presentaron sepsis (56\%), 3 pacientes neumonía (8\%), 1 perforación intestinal (3\%), 1 enterocolitis necrotizante (3\%), 1 choque séptico $(3 \%)$ y 10 pacientes de ese grupo no presentaron morbilidades $(27 \%)$.

En cambio, en los pacientes con cierre en dos tiempos quirúrgicos, 24 pacientes presentaron sepsis (51\%), 3 perforación intestinal (6\%), 3 oclusión intestinal $(6 \%)$, 2 enterocolitis necrotizante (4\%), 2 neumonía (4\%) y 13 pacientes no presentaron ninguna morbilidad. (29\%).

Cada uno de los grupos (los que presentaron morbilidades, los que fallecieron y los que no presentaron complicación alguna) se estratificó en cuatro subgrupos, tomando en cuenta el GPS; de los cuales existieron 60 pacientes que tuvieron morbilidades $(72 \%), 7$ pacientes fallecieron $(8 \%)$ y 16 pacientes no tuvieron complicación alguna (20\%), con una $p<0.05$ (Tabla 1).

Estratificando el grupo de morbilidades de acuerdo con el puntaje: en el grupo de 0-1 puntos hubo 20 pacientes, de los cuales 17 cursaron con sepsis (85\%), 2 con neumonía (10\%) y 1 con choque séptico $(5 \%)$. En el grupo de 2-3 puntos hubo 32 pacientes: a 24 se les diagnosticó con sepsis, 3 con enterocolitis necrotizante, 2 con oclusión intestinal, 2 con neumonía y solo 1 con perforación intestinal. En el grupo de 4-5 puntos hubo 7 pacientes, de los cuales 3 cursaron con sepsis, 3 con perforación intestinal y 1 con oclusión intestinal.
Tabla 1. Resultados del Gastroschisis Prognostic Score (GPS) en cada grupo de pacientes*

\begin{tabular}{|l|c|c|c|c|}
\hline GPS & Morbilidad & Mortalidad & OK & Total \\
\hline $0-1$ & 20 & 0 & 7 & 27 \\
\hline $2-3$ & 32 & 0 & 7 & 39 \\
\hline $4-5$ & 7 & 2 & 2 & 11 \\
\hline $6-7$ & 1 & 5 & 0 & 6 \\
\hline 8 & 0 & 0 & 0 & 0 \\
\hline Total & 60 & 7 & 16 & 83 \\
\hline
\end{tabular}

*Prueba exacta de Fischer: $27.12(p<0.05)$.

En el último grupo, de 6-7 puntos, solo se encontró 1 paciente y presentó neumonía. La asociación del puntaje con las complicaciones tuvo una $p<0.05$ (Tabla 2).

En contraste, en el grupo de mortalidad las causas se dividieron en choque séptico, síndrome de intestino corto, falla orgánica múltiple y necrosis intestinal. En el grupo de 4-5 puntos, 1 paciente murió de falla orgánica múltiple $(50 \%)$ y 1 paciente a consecuencia de necrosis intestinal $(50 \%)$. Los motivos de defunción de los pacientes que obtuvieron puntajes de 6-7 puntos fueron: 3 pacientes por choque séptico $(60 \%), 1$ con síndrome de intestino corto (20\%) y 1 con necrosis intestinal (20\%); cubriendo así los siete decesos del estudio. La asociación del puntaje con las causas de mortalidad tuvo una $\mathrm{p}<0.05$ (Tabla 3).

La media de la EIH fue de 36 días, con una DE de 25 y un rango de 0-195. En promedio los pacientes que presentaron cierre primario tuvieron 34 días de $\mathrm{EIH}$, mientras que los pacientes con cierre en dos tiempos tuvieron una media de 38 (Tabla 4).

Los pacientes que no tuvieron ninguna morbilidad 0 mortalidad tuvieron una media de EIH de 27, con DE de 8 y un rango entre 15 y 48 días. Los pacientes con morbilidad presentaron una media de 39 , DE de 27 , su mínimo fue de 15 y el máximo de 195 días. Mientras que los pacientes con mortalidad presentaron una media de 39 días de EIH, DE de 24 y un rango de 0 a 60 , con una $p$ de 0.140 (Tablas 5 y 6).

La media y los rangos de ElH en el grupo de morbilidad fueron las siguientes: en el puntaje de 0-1 tuvieron una media de 30 , un mínimo de 15 y un máximo de 60 días; en el grupo de 2-3 la media fue de 35 días, un mínimo de 17 y un máximo de 77; con puntaje de 4-5 la media fue de 42, con un rango de 15 a 107; y 
Tabla 2. Asociación del Gastroschisis Prognostic Score (GPS) con complicaciones

\begin{tabular}{|l|c|c|c|c|c|c|}
\hline GPS & Oclusión Intestinal & Sepsis & ECN & Perforación intestinal & Choque séptico & Neumonía \\
\hline $0-1$ & 0 & 17 & 0 & 0 & 1 & 2 \\
\hline $2-3$ & 2 & 24 & 3 & 1 & 0 & 2 \\
\hline $4-5$ & 1 & 3 & 0 & 3 & 0 & 0 \\
\hline $6-7$ & 0 & 0 & 0 & 0 & 0 & 1 \\
\hline Total & 3 & 44 & 3 & 4 & 1 & 5 \\
\hline
\end{tabular}

ECN: Enterocolitis necrosante.

Tabla 3. Asociación de puntaje con causas de mortalidad*

\begin{tabular}{|c|c|c|c|c|}
\hline GPS & $\begin{array}{c}\text { Choque } \\
\text { Séptico }\end{array}$ & $\begin{array}{c}\text { Síndrome de } \\
\text { intestino corto }\end{array}$ & $\begin{array}{c}\text { Falla orgánica } \\
\text { múltiple }\end{array}$ & $\begin{array}{c}\text { Necrosis } \\
\text { Intestinal }\end{array}$ \\
\hline $4-5$ & 0 & 0 & 1 & 1 \\
\hline $6-7$ & 3 & 1 & 0 & 1 \\
\hline Total & 3 & 1 & 1 & 2 \\
\hline
\end{tabular}

*Test exacto de Fisher: 36.457 ( $\mathrm{p}<0.05)$.

GPS: Gastroschisis Prognostic Score.

Tabla 4. Días de estancia intrahospitalaria (DEIH) por tipo de cierre*

\begin{tabular}{|l|c|c|c|}
\hline DEIH & Media & DE & Rangos \\
\hline Cierre primario & 33.58 & 31.16 & $0-195$ \\
\hline $\begin{array}{l}\text { Cierre en 2 } \\
\text { tiempos }\end{array}$ & 37.68 & 18.67 & $15-107$ \\
\hline
\end{tabular}

*U de Mann-Whitney $(\mathrm{p}=0.059)$.

DE: desviación estándar.

en el grupo de 6-7 tuvieron en promedio 56 días de ElH, con un rango de 0 a 195.

Respecto al grupo de mortalidad, en el grupo de 0-3 tuvo una media de 33 , con un rango de 15 a 77 días; en el grupo de 4-5 la media fue de 43 días, un mínimo de 15 y un máximo de 107; y en el de 6-7 la media fue de 53, con un rango de 0 a 195.

En los pacientes que evolucionaron satisfactoriamente sin complicaciones un puntaje menor a 5 no predice mayor EIH ( $\left.r^{2}: 0.000 ; p=0.953\right)$. En el grupo de mortalidad un puntaje mayor a 4 no predice mayor EIH ( $\left.r^{2}: 0.001 ; p=0.944\right)$. Por el contrario, en el grupo de morbilidad un puntaje menor a 5 de la escala GPS tiene mayor ElH ( $r^{2}: 0.223 ; p<0.05$; intervalo de confianza del 95\%: 9.341-27.33).

\section{Discusión}

La GPS se basa en cuatro criterios para valorar la lesión intestinal: adherencias intestinales (0 puntos = ausente, 1 = leve, 4 = severo), ausencia, sospecha o presencia de atresia intestinal $(0,1$ y 2 puntos respectivamente), necrosis intestinal (ausente $=0 \mathrm{y}$ presente $=4$ ) y perforación intestinal $(0=$ ausente $\mathrm{y}$ $2=$ presente $^{6}$.

Puligandla, et al. demuestran en su estudio que por cada punto que se incrementa en la GPS el paciente experimentará un incremento de su ElH de 16 días, mientras que en nuestro estudio los únicos que obtuvieron significancia estadística fueron los pacientes con morbilidad, demostrando que con un puntaje menor a 5 predice mayor $\mathrm{ElH}^{7}$.

Con GPS menores a 2 se considera que los pacientes son de bajo riesgo, mientras que los pacientes con puntajes $\geq 2$ se consideran de alto riesgo para morbilidad y los pacientes con puntajes $\geq 4$ tienen alto riesgo tanto para morbilidad como mortalidad, sin embargo, existen muchos pacientes que evolucionan satisfactoriamente independientemente del puntaje.

Steven, et al. realizaron un estudio en donde clasifican a los recién nacidos con gastrosquisis simple o compleja. La gastrosquisis compleja la definen como la presencia de atresia intestinal, perforación o vólvulos. En su estudio determinaron que el grupo de pacientes con gastrosquisis compleja se asocia con un incremento en los días de ElH comparados con los pacientes con gastrosquisis leve ${ }^{8}$. Así mismo, Shalaby, et al. mencionan que los pacientes con bajo riesgo tuvieron una menor $\mathrm{EIH}$; reportan una media de 14 días de EIH con un rango de 1-52 en una muestra de 24 pacientes ${ }^{9}$. Por otro lado, nuestro estudio tuvo una media de 36 días, con una DE de 25 y un rango de 0-195 en una muestra de 83 pacientes. 
Tabla 5. Media, desviación estándar (DE) y rangos de estancia intrahospitalaria (EIH) de cada grupo

\begin{tabular}{|l|c|c|c|c|c|c|c|c|c|}
\hline & \multicolumn{3}{|c|}{ OK } & \multicolumn{3}{c|}{ Morbilidad } & \multicolumn{3}{c|}{ Mortalidad } \\
\hline & Media & DE & Rangos & Media & DE & Rangos & Media & DE & Rangos \\
\hline ElH en días & 27 & 8 & $15-48$ & 39 & 27 & $15-195$ & 29 & 24 & $0-60$ \\
\hline
\end{tabular}

*Prueba Kruskal-Wallis: $3.927(p=0.140)$.

Tabla 6. Días de estancia intrahospitalaria (DEIH) acumulados por subgrupo

\begin{tabular}{|c|c|c|c|}
\hline GPS & OK (DEIH) & Morbilidad (DEIH) & Mortalidad (DEIH) \\
\hline $0-1$ & 179 & 643 & 0 \\
\hline $2-3$ & 205 & 1156 & 0 \\
\hline $4-5$ & 46 & 354 & 60 \\
\hline $6-7$ & 0 & 195 & 142 \\
\hline Total & 430 & 2348 & 202 \\
\hline
\end{tabular}

GPS: Gastroschisis Prognostic Score.

Existe muy poca literatura que relacione específicamente el GPS con los días de estancia intrahospitalaria. Nosotros demostramos que en el grupo de morbilidad existe significancia estadística al predecir que un puntaje menor a 5 indica mayor estancia intrahospitalaria; mientras que en el grupo de mortalidad y en los pacientes que evolucionaron de forma favorable no se demostró significancia estadística alguna.

A pesar de que el GPS toma en cuenta los puntajes ya mencionados para valorar la morbilidad y mortalidad en un paciente, con este estudio podemos mencionar que existen muchos pacientes que evolucionan satisfactoriamente de forma independiente del puntaje. Consideramos que la GPS no predice EIH en pacientes con mortalidad ni en los que evolucionan de una manera adecuada.

La limitante de este estudio muy probablemente fue el tamaño de muestra, a pesar de que fueron tomados en los principales centros hospitalarios de la ciudad de Puebla. Como comentamos anteriormente, el GPS solo maneja dos rubros: morbilidad y mortalidad. Tuvimos pacientes que evolucionaron satisfactoriamente independientemente del puntaje, por lo que una sugerencia es delimitar a los pacientes sin morbilidad. Lo ideal es que más investigadores se sumen a este propósito con la finalidad de incrementar la muestra y así determinar si en los demás pacientes es posible predecir su ElH con base en su puntaje.

\section{Financiamiento}

La presente investigación no ha recibido ninguna beca específica de agencias de los sectores públicos, comercial o sin ánimo de lucro.

\section{Conflicto de intereses}

Los autores declaran no tener conflicto de intereses alguno.

\section{Responsabilidades éticas}

Protección de personas y animales. Los autores declaran que para esta investigación no se han realizado experimentos en seres humanos ni en animales.

Confidencialidad de los datos. Los autores declaran que han seguido los protocolos de su centro de trabajo sobre la publicación de datos de pacientes.

Derecho a la privacidad y consentimiento informado. Los autores declaran que en este artículo no aparecen datos de pacientes.

\section{Bibliografía}

1. Salinas-Torres VM, Salinas-Torres RA, Cerda-Flores RM, Martínez-de-Villarreal LE. Prevalence, mortality, and spatial distribution of gastroschisis in Mexico. J Pediatr Adolesc Gynecol. 2018;31(3):232-7.

2. Zalles VC, Peñarrieta $D A$, Bracho BE, Ibarra RD, Dávila PR, Villegas $S R$, et al. A gastroschisis bundle: Effects of a quality improvement protocol on morbidity and mortality. Pediatr Surg. 2018;53(11).

3. Cowan KN, Puligandla PS, Laberge JM, Skarsgard ED, Bouchard S, Yanchar N, et al. The gastroschisis prognostic score: Reliable outcome prediction in gastroschisis. J Pediatr Surg. 2012;47(6):1111-7.

4. Calderón-Alvarado AB, Rojas-Villegas M del S, Dehesa-López E. Prevalencia de malformaciones congénitas detectadas al nacimiento en un hospital de segundo nivel en Sinaloa. Acta Pediatr Méx. 2017;38(6): 363-80

5. Sydorak RM, Nijagal A, Sbragia L, Hirose S, Tsao K, Phibbs RH, et al. Gastroschisis: Small hole, big cost. J Pediatr Surg. 2002;37(12):1669-72.

6. The Canadian Pediatric Surgery Network. CAPSNet 2019 Annual Report. Eng Technol. 2020;14(1):8-10.

7. Puligandla PS, Baird R, Skarsgard ED, Emil S, Laberge JM. Outcome prediction in gastroschisis - The gastroschisis prognostic score (GPS) revisited. J Pediatr Surg. 2017;52(5):718-21.

8. Raymond SL, Hawkins RB, St. Peter SD, Downard CD, Qureshi FG, Renaud $\mathrm{E}$, et al. Predicting morbidity and mortality in neonates born with gastroschisis. J Surg Res. 2020;245:217-24.

9. Shalaby A, Obeida A, Khairy D, Bahaaeldin K. Assessment of gastroschisis risk factors in Egypt. J Pediatr Surg. 2020;55(2):292-5. 